\title{
Correlación de los resultados de impacto o tenacidad de 16 maderas mexicanas utilizando dos métodos de prueba
}

\author{
Guadalupe Bárcenas Pazos ${ }^{1}$ \\ Reyna Paula Zárate Morales ${ }^{1}$ \\ Víctor Rubén Ordóñez Candelaria ${ }^{1}$ \\ Abigail Guerrero Báez ${ }^{1}$ \\ J. Amador Honorato Salazar ${ }^{2}$
}

RESUMEN

La resistencia de la madera al impacto se considera una propiedad mecánica de gran importancia, sobre todo para determinar la susceptibilidad de una madera para ser usada en mangos de herramientas o artículos deportivos, como mangos de pistola, bates de béisbol u otros usos en los que sea necesario absorber y disipar la energía de impacto. Existen varios métodos de ensayo para determinarla; entre los que se utilizan en México están el de Tenacidad basado en la norma ASTM D143 (ASTM, 1999) y el de Impacto o Flexión Dinámica basado en la Norma NF B51-009 (AFN, 1942). Cada tipo de ensayo presenta los resultados obtenidos con diferentes unidades. Con el fin de obtener la correlación de los resultados de ambos métodos de ensayo y así poder homologar los resultados ya existentes en la bibliografía sobre la resistencia al impacto de las maderas mexicanas, se realizaron ensayos con especímenes por pares para 16 maderas mexicanas, en condición seca y en condición saturada, con cada uno de los métodos. Se obtuvo una ecuación que permitirá comparar los resultados publicados en la bibliografía existente, con el fin de contar con un panorama más amplio del comportamiento de las maderas mexicanas ante las solicitaciones de resistencia al impacto.

PALABRAS CLAVE:

Flexión dinámica, impacto, maderas mexicanas, métodos de prueba, tenacidad.

\begin{abstract}
The wood impact bending resistance is considered an important mechanical property, mainly to determine the suitability of woods to be used for tool hammers or sport goods, such as pistol handles, base ball bats and other uses where it would be a need to absorb and to disperse energy during impact. There are several test methods to determine wood shock resistance, among those used in Mexico are the toughness test based on the ASTM D143 standard (ASTM, 1999) and the Impact or dynamic flexure test, based on the NF B51-009 standard (AFN, 1942). Each method produces values in different units. In order to find the correlation between the results from these two different test method to allow the comparison and homogenization of data already published on Mexican woods impact bending resistance, a testing program for 16 woods was carried out. Test were performed in green and dry conditions in both testing methods. An equation from a simple regression linear analysis was obtained comparing both sets of results. The equation will help to wide the scope of the knowledge on mechanical properties of Mexican woods.
\end{abstract}

KEY WORDS:

Dynamic bending, impact, Mexican woods, test methods, toughness. 


\section{INTRODUCCIÓN}

El impacto se puede definir como un choque repentino entre dos cuerpos. Un material con tenacidad alta es aquel que no falla abruptamente sino que su falla ocurre lentamente. Sin embargo, para la madera, el término tenacidad se ha descrito como una propiedad particular de ésta, medida estrictamente bajo la acción de una carga de impacto que sea capaz de provocar la falla. La tenacidad es el trabajo requerido para provocar la ruptura de un espécimen dado sometido a flexión mediante una carga de impacto (Bodig y Jayne, 1986).

La determinación de la tenacidad de la madera es importante por tres razones: a) determinar la variabilidad de la resistencia al choque de diferentes maderas, mediante el ensayo de especímenes provenientes de varios individuos de una misma especie; b) determinar la capacidad de la madera para ser utilizada en artículos que requieran ser capaces de absorber cargas de impacto aplicadas repetidamente, sin fallar p.e. mangos de herramienta, artículos deportivos como bates de béisbol y cachas de pistolas; c) para estimar la presencia de pudrición en algunos elementos (Brown et al., 1952; Jayne y Bodig, 1986).

La capacidad de la madera para absorber energía se puede determinar de diversas maneras. Un procedimiento consiste en calcular esta capacidad a partir de gráficas de esfuerzo-deformación obtenidas de pruebas de flexión estática. Otro método, el analizado en este trabajo, consiste en aplicar una carga repentina, al centro del claro, sobre una probeta en forma de viga simplemente apoyada. En este procedimiento se mide la energía absorbida por la probeta cuando se alcanza la fractura total de la pieza (Echenique y Robles, 1993).

En México, los datos de tenacidad publicados para algunas maderas que crecen en el país se han obtenido utilizando el método de aplicar la carga sobre una viga simplemente apoyada en sus extremos, basados en lo establecido, principalmente, por dos normas: la Norma ASTM D-143 (ASTM, 1998) y la Norma NF B51-009 (AFN, 1942). Los datos resultado de ensayos publicados por Novelo (1964), Echenique y Díaz (1969), Negrete (1970), Quiñónez (1974), Romero et al. (1982) y Fuentes (1990) se obtuvieron utilizando la primera de ellas, presentando W (Trabajo) en $\mathrm{kg}-\mathrm{m}$. Los datos publicados por Ordóñez et al. (1989), Bárcenas y Ortega (1993), Bárcenas (1995), Machuca (1995), Ordóñez et al. (1999) y Zárate et al. (2001) se obtuvieron utilizando la Norma ASTM, presentando $\mathrm{T}$ (tenacidad) en $\mathrm{kg}$ $\mathrm{cm} / \mathrm{cm}^{3}$.

La recopilación de la información sobre el comportamiento de las maderas mexicanas, con el fin de contar con un universo más completo de información sobre las propiedades mecánicas de éstas, no es posible debido a que los valores de tenacidad publicados y disponibles se presentan con diferentes las unidades, no permitiendo realizar una comparación entre los mismos.

\section{OBJETIVO}

Obtener una ecuación de correlación entre los resultados de impacto obtenidos con la Norma ASTM D-143 (ASTM, 1998) y los obtenidos con la Norma NF B51-009, (AFN, 1942), realizando ensayos con especímenes pares (matched specimens), a dos contenidos de humedad, de la madera de 16 especies forestales que crecen en México.

\section{TENACIDAD DE LA MADERA}

La tenacidad de la madera, como se menciona párrafos arriba, se define como su capacidad para absorber la energía de un choque. Es una propiedad mecánica y como tal está afectada por las características propias del material, es decir presenta una variabilidad dependiendo del tipo de madera que se ensaya, su 
contenido de humedad $(\mathrm{CH})$, la orientación de los anillos de crecimiento y la dirección de la fibra, entre otros.

En general la resistencia mecánica de la madera, a medida que disminuye el contenido de humedad, se incrementa debido a la densificación y rigidización de las paredes de las células y al aumento de material leñoso por unidad de volumen, originado por la contracción ocasionada por la pérdida de agua (Echenique y Robles, 1993). La resistencia al impacto y la tenacidad de la madera, presentan un efecto contrario a la regla de los cambios de resistencia en las demás propiedades mecánicas de la misma, debido a las variaciones del contenido de humedad $(\mathrm{CH})$. Ambas propiedades de impacto representan el trabajo que realiza una pieza de madera, esto es, el producto de una carga aplicada multiplicada por la deformación del espécimen y son la combinación del esfuerzo de flexión y de la plasticidad de la madera. Esta última se incrementa directamente en proporción al incremento del contenido de humedad por arriba del 30\% (punto de saturación de la fibra, PSF), y controla el comportamiento de la tenacidad en las propiedades tanto para flexión por impacto como estática.

Panshin y DeZeew (1980) establecen que los valores de tenacidad, cuando son obtenidos mediante un ensayo de flexión por impacto, tienen variaciones del orden de $0.5 \%$ a $1 \%$, como respuesta ante los cambios de contenido de humedad por cada incremento por ciento de éste. Echenique y Robles (1993) mencionan a su vez, que la tenacidad determinada en madera libre de defectos no se ve incrementada conforme disminuye el contenido de humedad, debido a que cuando está verde, la madera absorbe, en general, mayor energía que cuando está seca, aunque el esfuerzo máximo que puede alcanzar sea menor.

Los defectos que tienen mayor influencia en el comportamiento de la madera sujeta a cargas de impacto son: la desviación de fibra y la presencia de hongos manchadores. La anisotropía de la madera, a su vez, afecta de manera considerable sus características físicomecánicas, es decir, su comportamiento ante diferentes acciones presenta diferencias, dependiendo de la dirección en que éstas se apliquen. En el caso de la tenacidad, generalmente presenta valores más altos en dirección tangencial que en la radial (FPL, 1999).

La duración de la carga es de gran importancia durante la determinación de las propiedades mecánicas de la madera, debido a que su resistencia se ve afectada en mayor o menor escala, dependiendo del tiempo que dure la aplicación de la carga, es decir, la resistencia de la madera disminuye considerablemente con una duración de carga prolongada y cuando la duración de la carga es corta, el valor de resistencia que alcanza es mayor. En general, la madera presenta altos valores de resistencia ante cargas de impacto.

\section{MÉTODOS DE ENSAYO}

Norma ASTM. Un método para determinar la tenacidad dela madera es un ensayo a flexión por medio de un sólo impacto; los resultados obtenidos en este tipo de método dependen del tamaño del espécimen ensayado y se expresan como el trabajo realizado al fracturar la probeta, no pueden ser fácilmente comparados con otros resultados por lo que deben ser manejados cuidadosamente (Bodig y Jayne, 1986). La tenacidad de la madera puede ser definida como su capacidad para absorber la energía de un impacto y el método de prueba se encuentra establecido en la Norma ASTM D-143 (ASTM, 1998).

La máquina para determinar la tenacidad de la madera fue diseñada por el Laboratorio de Productos Forestales de Madison, Wisconsin del Departamento de Agricultura de los Estados Unidos (FPLUSDA) (Fig. 1). Esta máquina es de 
impacto, diseñada de tal manera que la aplicación súbita de la carga no se realice por la caída de un martillo sino a través de un cable de acero flexible sujeto a un péndulo sobre el centro de la probeta apoyada en dos pernos de reacción alineados verticalmente (Brown et al., 1952, Bodig y Jayne, 1986). El ángulo inicial del péndulo se puede fijar en $30^{\circ}$, $45^{\circ}$ ó $60^{\circ}$; la diferencia que se registra entre el ángulo inicial seleccionado y el ángulo final se utiliza para calcular la energía requerida para provocar la ruptura total del espécimen, será un índice de la tenacidad (T) de la probeta (Brown et al., 1952).

La norma ASTM D-143 (ASTM, 1998) establece que los ensayos se realicen con dos lotes: en uno aplicando la carga en la cara radial y otro aplicando la carga en la cara tangencial con dirección radial, es decir, la inclinación de los anillos

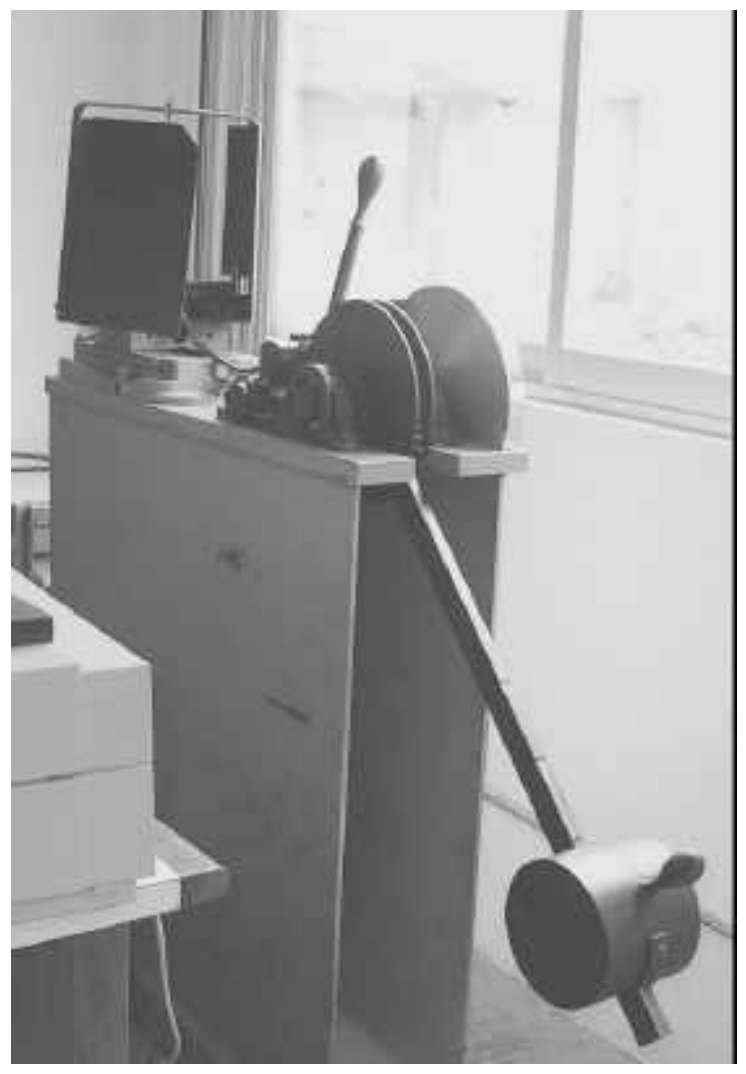

Figura 1. Máquina para ensayos de Tenacidad por impacto (Modelo FPL). debe ser de $0^{\circ}$ y $90^{\circ}$ en cada lote, respectivamente (Fig. 2). Sin embargo, los datos publicados resultados de ensayos realizados bajo esta Norma fueron realizados únicamente en la dirección radial, sobre la cara tangencial más cercana a la médula, por ser ésta lo condición más desfavorable para la madera cuando es sujeta a cargas en flexión (FPL, 1999).

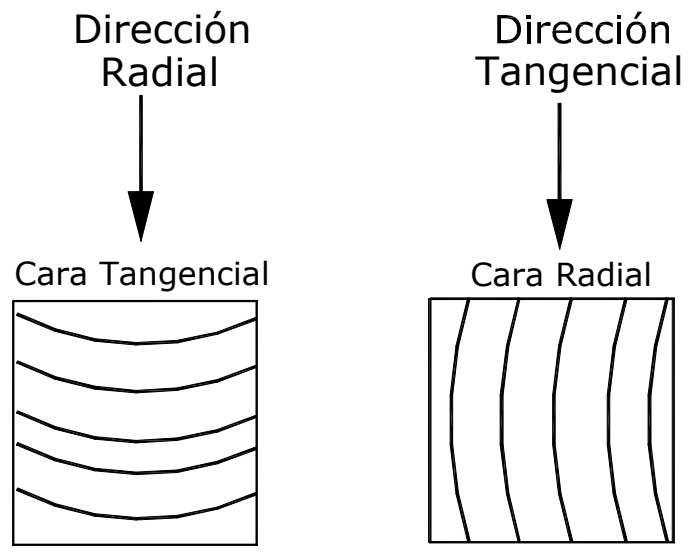

Figura 2. Condiciones de aplicación de la carga

En la Norma ASTM (ASTM D-143) se establece el cálculo de la tenacidad utilizando la ecuación

$$
T=M(\cos \phi-\cos \theta)
$$

donde:

$$
\begin{aligned}
T= & \text { tenacidad } \\
M= & \text { momento del péndulo alrededor } \\
& \text { del eje de rotación } \\
\phi= & \text { ángulo final del péndulo } \\
\theta= & \text { ángulo inicial del péndulo }
\end{aligned}
$$

El valor numérico de $M$ depende del peso de la barra, del peso del péndulo y del cilindro sin incluir la cadena, de la distribución del peso con referencia el eje de rotación, de la gravedad, de la ubicación de la barra del péndulo y del peso de la clavija para fijar la barra, es un valor 
constante especificado por el fabricante de la máquina. El ángulo inicial es nominal, puede ser 30, 45 ó $60^{\circ}$ y el ángulo final es el que se registra al momento de fallar el espécimen de prueba.

Norma NF. El método de prueba de Impacto o Flexión Dinámica, establecido en la NF B51-009 (AFN, 1942), se realiza con la máquina Amsler (Fig. 3). Este método consiste en romper una probeta dispuesta como viga simplemente apoyada a la que se aplica una carga súbita al centro del claro con un péndulo que se deja oscilar desde una altura determinada, de forma similar al golpe de un martillo.

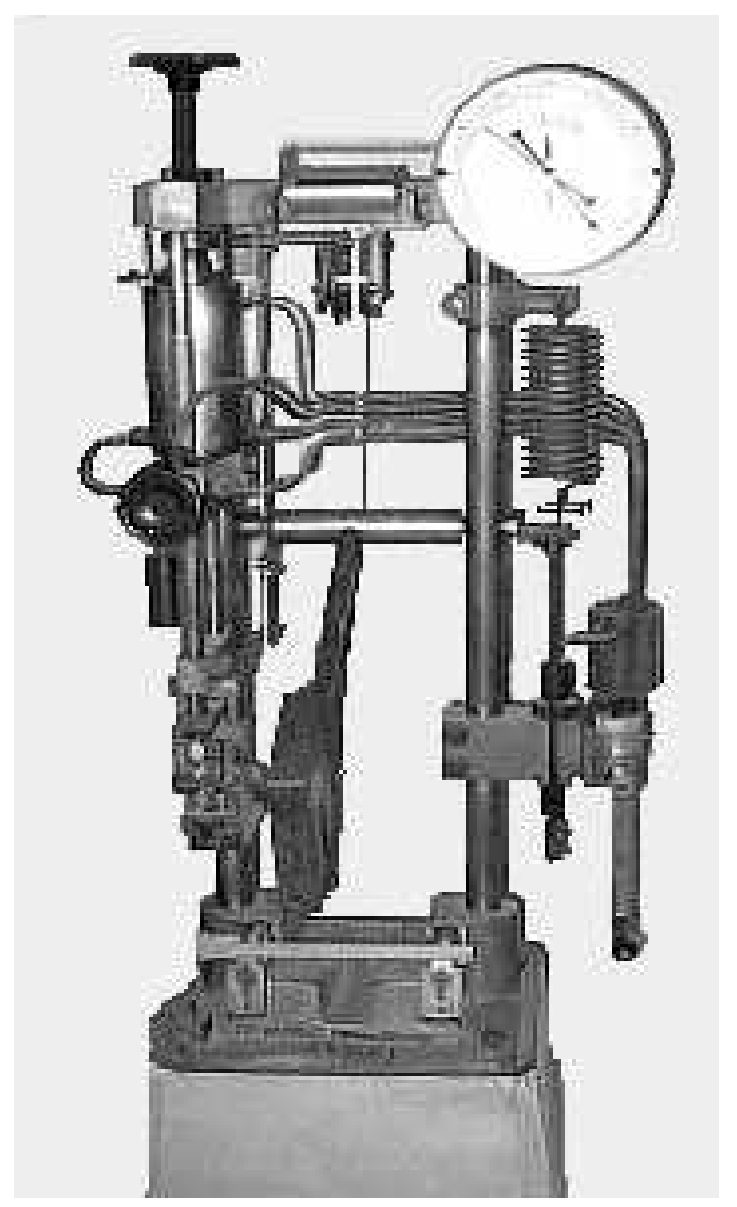

Figura 3 . Máquina para ensayos de impacto o Flexión dinámica.
Con este método se evalúa la energía que es capaz de absorber la madera cuando se somete a cargas de impacto (golpe súbito). A la energía absorbida se denomina Trabajo de ruptura (W), también se obtienen los valores de Reacción instantánea $(R)$, Coeficiente de resiliencia (k), Cota dinámica (Cd) y Densidad normal (Dn) utilizada para el cálculo de la cota dinámica, usando las ecuaciones 1, 2, 3 y 4 . El valor de la energía absorbida por la probeta se mide directamente de la máquina AMSLER en la escala de potencia de la máquina, con una precisión de $0.1 \mathrm{~kg}-\mathrm{m}$; según lo indicado en sus especificaciones de fabricación. El valor W no se afecta por algún tipo de constante, ni se utiliza expresión matemática alguna para obtenerlo.

$$
\begin{gathered}
R=F H \\
k=\frac{W}{b d^{10 / 6}} \\
C d=\frac{k}{D n^{2}} \\
D n=\frac{P_{\text {prob }}}{V_{\text {prob }}}
\end{gathered}
$$

donde:

$$
\begin{aligned}
b= & \text { grueso, } \mathrm{cm} \\
C d= & \text { cota dinámica } \\
d= & \text { peralte }, \mathrm{cm} \\
D n= & \text { Densidad normal } \\
\mathrm{F}= & \text { Exp }(-0.24475+2.02595 * \mathrm{LN} \\
& (\varnothing)), \text { valor correspondiente al } \\
& \text { diámetro de impresión ( }(\varnothing) \text { en la } \\
& \text { barra de aluminio, }(\mathrm{kg}) \\
H= & \text { Dureza de la barra de aluminio de } \\
& 34.6 \\
= & \text { Coeficiente de resiliencia } \\
\mathrm{P}_{\text {prob }}= & \text { Peso de la probeta } \\
R= & \text { Reacción instantánea, } \mathrm{kgf} \\
V_{\text {prob }}= & \text { Volumen de la probeta } \\
W= & \text { Trabajo de ruptura kg-m. }
\end{aligned}
$$


La norma NF B51-009 (ANF, 1942) establece que el esfuerzo debe ser ejercido en la dirección tangencial de la probeta, es decir, la carga debe ser aplicada en su cara radial con inclinación de los anillos de $0^{\circ}$ con respecto a la carga aplicada.

De la descripción de los dos métodos se observa que el resultado obtenido es energía, pero para la determinación del valor del trabajo (W), no se considera el volumen del espécimen mientras que en el cálculo de la tenacidad si se utiliza. De ahí la importancia de hallar un factor de correlación que permita convertir los resultados publicados obtenidos por cada uno de los métodos y lograr un grupo de información más completo sobre la capacidad de la madera para absorber energía ante cargas por impacto.

\section{MÉTODOLOGÍA}

Selección de material. Se seleccionó material proveniente de 16 especies maderables, colectadas con anterioridad en tres diferentes estados de la República mexicana y pertenecientes todas al grupo de las angiospermas, tanto templadas como tropicales. Las especies estudiadas, el número de árboles por especie y el número de probetas por condición y por método ensayado se presentan en la Tabla 1. El material seleccionado fue libre de defectos: sin desviación en la fibra, presencia de nudos o grietas, ataque de hongos manchadores, pudrición o insectos. El material estaba secado al aire libre.

Tabla 1. Especies, procedencia, número de árboles y de probetas ensayadas

\begin{tabular}{|c|c|c|c|c|c|c|}
\hline \multirow{3}{*}{$\begin{array}{l}\text { ESPECIE } \\
\text { Ampelocera hottlei (Standl.) Standl. }\end{array}$} & \multirow{3}{*}{$\begin{array}{l}\text { PROCE- } \\
\text { DENCIA } \\
\text { Chis. (SAP) }\end{array}$} & \multirow{3}{*}{$\begin{array}{l}\text { NO. DE } \\
\text { ÁRBOLES } \\
2\end{array}$} & \multicolumn{4}{|c|}{ MÉTODO / CONDICIÓN } \\
\hline & & & \multicolumn{2}{|c|}{ ASTM } & \multicolumn{2}{|c|}{ NF } \\
\hline & & & 8 & 8 & 7 & 8 \\
\hline Bucida buceras L. & Q. Roo (SMP) & 1 & 4 & 3 & 4 & 4 \\
\hline Cedrela odorata L. & Chis. (SAP) & 2 & 7 & 7 & 6 & 6 \\
\hline Cosmocalyx spectabilis Standl. & Q. Roo (SMP) & 2 & 8 & 7 & 8 & 8 \\
\hline Guarea chichon C. DC. & Chis. (SAP) & 2 & 6 & 8 & 8 & 8 \\
\hline Getarda combsii Urb. & Q. Roo (SMP) & 2 & 8 & 8 & 8 & 8 \\
\hline Lonchocarpus castilloi Standl. & Chis. (SAP) & 1 & 4 & 4 & 3 & 3 \\
\hline Lysiloma acapulcense (Kunth) Benth & Chis. (SAP) & 2 & 8 & 8 & 8 & 8 \\
\hline Lysiloma bahamensis Benth. & Q.Roo (SMP) & 1 & 3 & 4 & 4 & 4 \\
\hline Magnolia schideana Schltdl. & Tams. (BMM) & 2 & 6 & 7 & 8 & 7 \\
\hline Metopium brownei (Jacq.) Urban & Q. Roo (SMP) & 1 & 4 & 3 & 4 & 4 \\
\hline Mirandaceltis monoica (Hems.) Sharp & Chis. (SAP) & 2 & 8 & 7 & 8 & 8 \\
\hline Quercus eugeniifolia Liebm & Ver. (BMM) & 1 & 4 & 4 & 4 & 4 \\
\hline Quercus mexicana Bonpl. & Ver. (BMM) & 1 & 4 & 4 & 4 & 4 \\
\hline Quercus uxoris McVaugh & Ver. (BMM) & 1 & 4 & 4 & 4 & 4 \\
\hline Vatairea lundellii (Standl.) killip. ex Record & Chis. (SAP) & 2 & 7 & 8 & 7 & 8 \\
\hline $\begin{array}{ll}\text { SAP } & \text { Selva alta perennifolia } \\
\text { SMP } & \text { Selva mediana perennifolia } \\
\text { BMM } & \text { Bosque mesófilo de montaña }\end{array}$ & En & el & $d e$ & & & \\
\hline
\end{tabular}


Diferencias con respecto a las Normas. Se realizaron dos modificaciones a lo establecido en las normas con el fin de obtener resultados susceptibles de ser comparados, teniendo presente que el objetivo del trabajo es obtener un factor de correlación entre los resultados ya publicados, obtenidos con los dos métodos. En el caso de la Norma NF B51009 se realizaron los ensayos en condición verde y seca, aun cuando la Norma sólo establece ensayos en condición seca; también se determinó la densidad relativa básica (peso anhídro/ volumen verde) para cada espécimen para efectos de comparación con los valores que se obtuvieron por el método establecido en la Norma ASTM D-143. En el caso de esta norma el material se ensayó aplicando la carga en la dirección radial, en la cara tangencial más cercana a la médula, por ser esta la condición más desfavorable de la madera cuando es sujeta a cargas de flexión.

Preparación de especímenes. Cada probeta fue identificada con una clave: por árbol, condición y método de ensayo. Las piezas de las que se obtendrían las probetas para ser ensayadas en condición seca se colocaron en una cámara de acondicionamiento con temperatura de $23^{\circ} \mathrm{C} \pm 3^{\circ} \mathrm{C}$ y humedad relativa de $65 \%$ $\pm 1 \%$, hasta alcanzar un contenido de humedad cercano al $12 \%$, como lo establece la norma ASTM D-143 (ASTM, 1999). El material a ensayarse en condición "verde" se colocó en una pileta con agua que las cubría totalmente hasta que alcanzó la condición saturada, es decir, un contenido de humedad por arriba del $30 \%$.

Una vez acondicionado el material, tanto en condición seca como en "verde", se elaboraron los especímenes de prueba. El tamaño final de las probetas ensayadas con el método que establece la Norma ASTM D-143 (ASTM, 1998) fue de $19 \mathrm{~mm}$ x $19 \mathrm{~mm}$ por $280 \mathrm{~mm}$. Para el lote ensayado con el método de la norma NF B51-009 (ANF, 1942) los especímenes fueron dimensionados con una sección transversal de $20 \mathrm{~mm} \times 20 \mathrm{~mm}$ y una longitud de $300 \mathrm{~mm}$.

Realización de ensayos. Para las probetas que se ensayaron utilizando la Norma ASTM, se hicieron ensayos previos para determinar el comportamiento de cada especie y así determinar qué especies se ensayarían con cada uno de los posibles ángulos iniciales de $60^{\circ}, 45^{\circ}$ ó $30^{\circ}$ y con qué posición del peso del péndulo en el brazo de palanca se debería utilizar para lograr la falla total. Se determinó que el material de las especies Cedrela odorata y Lysiloma acapulcense se ensayaría con un ángulo inicial de $45^{\circ}$ y posición 2 del peso del péndulo; la probetas de Cosmocalyx spectabilis se ensayarían con un ángulo inicial de $45^{\circ}$ y posición 1 del peso del péndulo; los especímenes de las 13 especies restantes se ensayarían con un ángulo inicial de $60^{\circ}$ y posición 1 del peso del péndulo.

Los basados en la Norma ASTM se efectuaron en el Departamento de Productos Forestales y Conservación de Bosques del Instituto de Ecología, A.C. en Xalapa, Ver. mientras que los basados en la Norma NF B51-009, se llevaron a cabo en las instalaciones del CENID-CITEMA del INIFAP en San Martinito Tlahuapa, estado de Puebla.

Las fallas se clasificaron de acuerdo con la forma en que se desarrollaron: falla astillada; falla por desviación de fibra, falla en astillas, falla escobillada y falla frágil o vidriosa, estos dos últimos tipos se desechan, si se debe a manchado por pudrición. Los tipos de falla se ilustran en la figura 4 (INECOL, 2000).

Cálculo de resultados. Para cada ensayo y por cada método y condición por especie se obtuvieron las magnitudes correspondientes con las ecuaciones 1 a 5. Para cada probeta por condición y método se determinó su contenido de humedad al momento de la prueba $(\mathrm{CH})$ y su densidad relativa básica (sobre las 


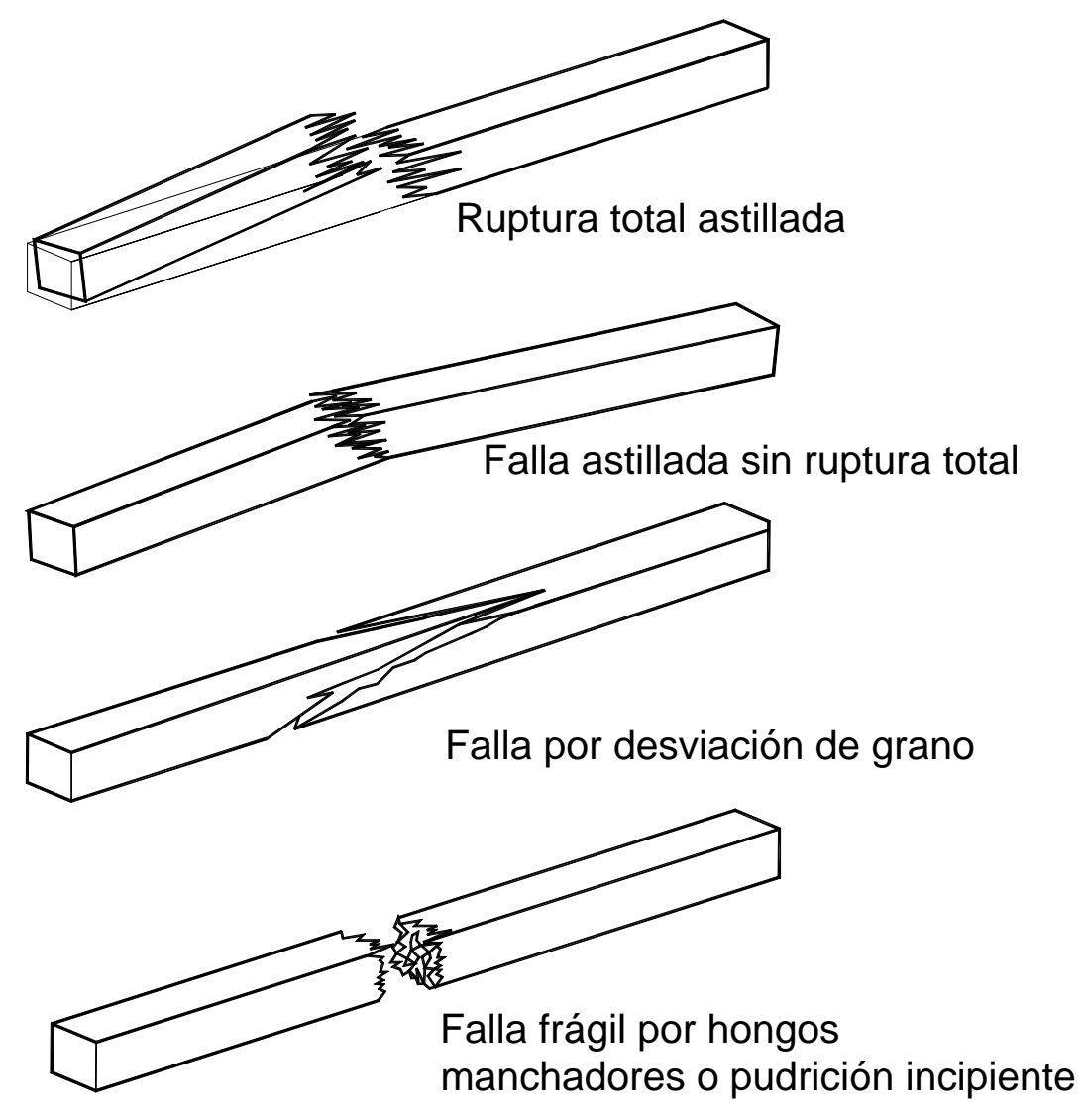

Figura 4. Tipos de falla por en ensayos de impacto (INECOL, 2000)

bases de peso anhidro y volumen verde) (DR). Para calcularlos, se obtuvo una porción de cada espécimen, de $50 \mathrm{~mm}$ de longitud, cercana a la falla pero sin contenerla. Inmediatamente después de cortada, dicha porción se pesó, cuidando que entre el corte y el pesado, se mantuviera en bolsas de plástico para evitar que se modificara su contenido de humedad.

Para determinar la densidad relativa básica en las probetas ensayadas en condición verde, se midió su volumen verde inmediatamente después de los ensayos, mientras que para las que se ensayaron al $12 \%$ de $\mathrm{CH}$, la porción fue saturada por dos semanas, cuando se registró el volumen verde. Después de que el total de las porciones se secaron en un horno durante 48 horas a una temperatura de $103{ }^{\circ} \mathrm{C} \pm 3{ }^{\circ} \mathrm{C}$, para obtener su peso anhidro. El valor final de la densidad calculada sobre las bases de peso anhidro y volumen verde se obtuvo con el promedio del total de probetas ensayadas en los dos métodos de prueba por cada especie.

Se calcularon los estadísticos básicos de promedio (media), desviación estándar (d.e.) y coeficiente de variación (c.v.) para cada especie, por condición y método de ensayo.

Los datos se obtuvieron de tal manera que coincidieran en las unidades con los que se encuentran ya publicados para que fuera posible aplicar el factor que se pretendía obtener para cada uno de los grupos de datos. 
Se realizó un análisis de regresión para encontrar un factor de correlación entre los resultados obtenidos por ambos métodos (NF B51-009, 1942 y ASTM D 143, 1999), graficando los valores de $W$, $(\mathrm{kg}-\mathrm{cm})$, contra los valores de $\mathrm{T}$ (kg $\mathrm{cm} / \mathrm{cm}^{3}$ ) tanto en condición seca como en condición verde. Se realizaron cuatro tipos de ajustes de curvas (lineal, potencial, logarítmico y exponencial); la curva que mejor se ajustó fue la potencial obteniéndose con ésta el coeficiente de determinación $\left(R^{2}\right)$ más alto. Este valor correspondió al ajuste potencial de la forma:

$$
Y=b x^{e}
$$

Donde:

$Y=$ variable dependiente $($ Tenacidad $T)$

$b=$ constante

$x=$ variable independiente (Trabajo de ruptura, W)

\section{RESULTADOS}

Los resultados obtenidos, valores promedio, desviación estándar y coeficiente de variación de cada magnitud se presentan en las Tablas 2, 3 y 4 . Las especies están ordenadas de acuerdo con su densidad relativa básica en forma ascendente.

Los resultados correspondientes a las pruebas de Tenacidad, realizadas con base en la Norma ASTM D-143, se presentan en la Tabla 4, valores de tenacidad ( $T$ ), en las dos condiciones de humedad. Los resultados obtenidos utilizando la Norma NF incluyen los valores promedio para cinco magnitudes: trabajo de ruptura (W, kg-m) (Tabla 2); coeficiente de resiliencia $(k)$, reacción instantánea $(\mathrm{R})$, cota dinámica $(\mathrm{Cd})$ (Tabla 3) en los dos contenidos de humedad establecidos. Para cada tabla se presentan el valor de contenido de humedad en condición seca y los de densidad relativa básica (peso anhidro/ volumen verde).

En las figuras 5 y 6 se presentan los resultados obtenidos por método de prueba y condición de contenido de humedad comparados con la densidad relativa básica (pa/vv). En las figuras 7 y 8 se presentan las graficas de correlación entre los valores de trabajo de los dos métodos ensayados, con sus correspondientes ecuaciones y factores de correlación para cada condición de humedad.

\section{DISCUSIÓN Y CONCLUSIONES}

Las tendencias obtenidas de los análisis de correlación en las dos condiciones de prueba son similares $R^{2}=$ 0.83 para la condición seca $( \pm 12 \% \mathrm{CH})$ y de $R^{2}=0.84$ para la condición seca, coeficientes de determinación altos, por lo que la ecuación obtenida se puede considerar adecuada para comparar los resultados obtenidos en la determinación de las propiedades de la madera para absorber energía, por cualquiera de los dos métodos ensayados. Resultará también útil para incrementar el universo del conocimiento de las propiedades de las madera mexicanas al ser posible convertir los datos publicados a unidades similares.

De los resultados obtenidos, aun cuando la muestra es pequeña para servir como índice de la tenacidad de la madera, sí mostró tendencias impor-tantes en el comportamiento de la madera en esta propiedad. Una de ellas es el efecto del contenido de humedad y la dirección de aplicación de la carga.

Las diferencias de los valores de la pendiente entre las correlaciones que se obtuvieron para la condición seca y condición verde puede atribuirse a que la propiedad mecánica de impacto es errática en su respuesta ante cambios de 
Tabla 2. Resultados de Flexión Dinámica de 16 especies mexicanas

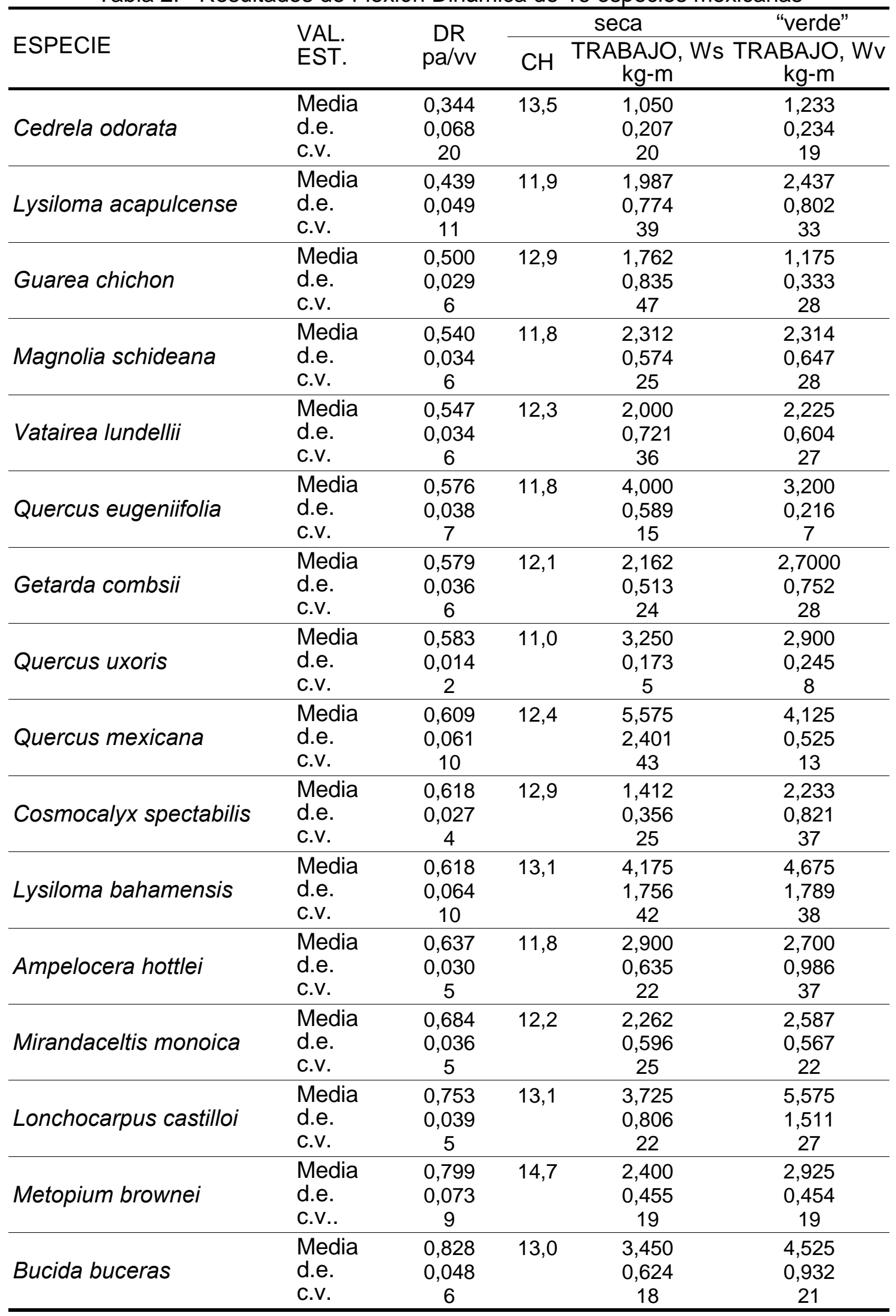


Tabla 3. Resultados Flexión Dinámica de 16 especies mexicanas

\begin{tabular}{|c|c|c|c|c|c|c|c|}
\hline \multirow[b]{2}{*}{ ESPECIE } & \multirow{2}{*}{$\begin{array}{l}\text { VAL. } \\
\text { EST. }\end{array}$} & \multicolumn{3}{|c|}{ CONDICIÓN SECA } & \multicolumn{3}{|c|}{ CONDICIÓN "VERDE" } \\
\hline & & $\mathrm{k}$ & $\begin{array}{c}\mathrm{R} \\
(\mathrm{kg})\end{array}$ & $\mathrm{Cd}$ & k & $\underset{(\mathrm{kg})}{\mathrm{R}}$ & $\mathrm{Cd}$ \\
\hline Cedrela odorata & $\begin{array}{l}\text { Media } \\
\text { d.e. } \\
\text { c.v. }\end{array}$ & $\begin{array}{c}0,166 \\
0,032 \\
19\end{array}$ & $\begin{array}{c}108,2 \\
12,6 \\
12\end{array}$ & $\begin{array}{c}0,858 \\
0,117 \\
14\end{array}$ & $\begin{array}{c}0,192 \\
0,037 \\
19\end{array}$ & $\begin{array}{c}112,0 \\
47,8 \\
43\end{array}$ & $\begin{array}{c}0,425 \\
0,091 \\
21\end{array}$ \\
\hline Lysiloma acapulcensis & $\begin{array}{l}\text { Media } \\
\text { d.e. } \\
\text { c.v. }\end{array}$ & $\begin{array}{c}0,313 \\
0,121 \\
39\end{array}$ & $\begin{array}{c}151,8 \\
28,5 \\
19\end{array}$ & $\begin{array}{c}1,234 \\
0,306 \\
25\end{array}$ & $\begin{array}{c}0,381 \\
0,129 \\
34\end{array}$ & $\begin{array}{c}143,5 \\
42,0 \\
29\end{array}$ & $\begin{array}{c}0,616 \\
0,088 \\
14 \\
\end{array}$ \\
\hline Guarea chichon & $\begin{array}{l}\text { Media } \\
\text { d.e. } \\
\text { c.v. }\end{array}$ & $\begin{array}{c}0,302 \\
0,168 \\
56\end{array}$ & $\begin{array}{c}171,4 \\
41,0 \\
24\end{array}$ & $\begin{array}{c}0,691 \\
0,249 \\
36\end{array}$ & $\begin{array}{c}0,181 \\
0,051 \\
28\end{array}$ & $\begin{array}{c}138,3 \\
31,4 \\
23\end{array}$ & $\begin{array}{c}0,230 \\
0,048 \\
21\end{array}$ \\
\hline Magnolia schideana & $\begin{array}{l}\text { Media } \\
\text { d.e. } \\
\text { c.v. }\end{array}$ & $\begin{array}{c}0,365 \\
0,092 \\
25\end{array}$ & $\begin{array}{c}187,1 \\
21,4 \\
11\end{array}$ & $\begin{array}{c}0,825 \\
0,193 \\
23\end{array}$ & $\begin{array}{c}0,372 \\
0,098 \\
26\end{array}$ & $\begin{array}{c}157,5 \\
39,2 \\
25\end{array}$ & $\begin{array}{c}0,356 \\
0,090 \\
25\end{array}$ \\
\hline Vatairea Iundellii & $\begin{array}{l}\text { Media } \\
\text { d.e. } \\
\text { c.v. }\end{array}$ & $\begin{array}{c}0,315 \\
0,113 \\
36\end{array}$ & $\begin{array}{c}175,6 \\
18,9 \\
11\end{array}$ & $\begin{array}{c}0,716 \\
0,257 \\
36\end{array}$ & $\begin{array}{c}0,347 \\
0,093 \\
27\end{array}$ & $\begin{array}{c}149,6 \\
49,3 \\
33\end{array}$ & $\begin{array}{c}0,318 \\
0,069 \\
23\end{array}$ \\
\hline Quercus eugeniifolia & $\begin{array}{l}\text { Media } \\
\text { d.e. } \\
\text { c.v. }\end{array}$ & $\begin{array}{c}0,629 \\
0,097 \\
15\end{array}$ & $\begin{array}{c}210,8 \\
10,2 \\
5\end{array}$ & $\begin{array}{c}1,147 \\
0,104 \\
9\end{array}$ & $\begin{array}{c}0,499 \\
0,043 \\
9\end{array}$ & $\begin{array}{c}197,0 \\
100,2 \\
51\end{array}$ & $\begin{array}{c}0,491 \\
0,028 \\
6\end{array}$ \\
\hline Getarda combsii & $\begin{array}{l}\text { Media } \\
\text { d.e. } \\
\text { c.v. }\end{array}$ & $\begin{array}{c}0,341 \\
0,077 \\
23\end{array}$ & $\begin{array}{c}199,2 \\
19,6 \\
10\end{array}$ & $\begin{array}{c}0,711 \\
0,235 \\
33\end{array}$ & $\begin{array}{c}0,418 \\
0,117 \\
28\end{array}$ & $\begin{array}{c}167,1 \\
24,2 \\
14\end{array}$ & $\begin{array}{c}0,368 \\
0,105 \\
28\end{array}$ \\
\hline Quercus uxoris & $\begin{array}{l}\text { Media } \\
\text { d.e. } \\
\text { c.v. }\end{array}$ & $\begin{array}{c}0,498 \\
0,042 \\
9\end{array}$ & $\begin{array}{c}220,6 \\
12,8 \\
6 \\
\end{array}$ & $\begin{array}{c}0,945 \\
0,083 \\
9\end{array}$ & $\begin{array}{c}0,462 \\
0,048 \\
10\end{array}$ & $\begin{array}{c}131,6 \\
46,2 \\
35\end{array}$ & $\begin{array}{c}0,421 \\
0,050 \\
12\end{array}$ \\
\hline Quercus mexicana & $\begin{array}{l}\text { Media } \\
\text { d.e. } \\
\text { c.v. }\end{array}$ & $\begin{array}{c}0,873 \\
0,377 \\
43\end{array}$ & $\begin{array}{c}256,5 \\
32,8 \\
13\end{array}$ & $\begin{array}{c}1,272 \\
0,348 \\
27\end{array}$ & $\begin{array}{c}0,656 \\
0,087 \\
13\end{array}$ & $\begin{array}{c}234,7 \\
82,0 \\
35\end{array}$ & $\begin{array}{c}0,594 \\
0,085 \\
14\end{array}$ \\
\hline Cosmocalyx spectabilis & $\begin{array}{l}\text { Media } \\
\text { d.e. } \\
\text { c.v. }\end{array}$ & $\begin{array}{c}0,272 \\
0,069 \\
25\end{array}$ & $\begin{array}{c}193,0 \\
23,9 \\
12\end{array}$ & $\begin{array}{c}0,495 \\
0,105 \\
21\end{array}$ & $\begin{array}{c}0,427 \\
0,157 \\
37\end{array}$ & $\begin{array}{c}157,8 \\
26,0 \\
16\end{array}$ & $\begin{array}{c}0,495 \\
0,227 \\
46\end{array}$ \\
\hline Lysiloma bahamensis & $\begin{array}{l}\text { Media } \\
\text { d.e. } \\
\text { c.v. }\end{array}$ & $\begin{array}{c}0,658 \\
0,276 \\
42\end{array}$ & $\begin{array}{c}223,4 \\
67,8 \\
30\end{array}$ & $\begin{array}{c}1,214 \\
0,474 \\
39\end{array}$ & $\begin{array}{c}0,716 \\
0,275 \\
38\end{array}$ & $\begin{array}{c}179,0 \\
11,2 \\
6\end{array}$ & $\begin{array}{c}0,623 \\
0,194 \\
31\end{array}$ \\
\hline Ampelocera hottlei & $\begin{array}{l}\text { Media } \\
\text { d.e. } \\
\text { c.v. }\end{array}$ & $\begin{array}{c}0,460 \\
0,102 \\
22 \\
\end{array}$ & $\begin{array}{c}141,1 \\
99,1 \\
70 \\
\end{array}$ & $\begin{array}{c}0,719 \\
0,212 \\
30\end{array}$ & $\begin{array}{c}0,420 \\
0,156 \\
37\end{array}$ & $\begin{array}{c}187,4 \\
49,9 \\
27\end{array}$ & $\begin{array}{c}0,359 \\
0,131 \\
36\end{array}$ \\
\hline Mirandaceltis monoica & $\begin{array}{l}\text { Media } \\
\text { d.e. } \\
\text { c.v. }\end{array}$ & $\begin{array}{c}0,356 \\
0,090 \\
25\end{array}$ & $\begin{array}{c}223,8 \\
28,2 \\
13\end{array}$ & $\begin{array}{c}0,492 \\
0,137 \\
28\end{array}$ & $\begin{array}{c}0,400 \\
0,084 \\
21\end{array}$ & $\begin{array}{c}190,7 \\
15,5 \\
8\end{array}$ & $\begin{array}{c}0,333 \\
0,049 \\
15\end{array}$ \\
\hline Lonchocarpus castilloi & $\begin{array}{l}\text { Media } \\
\text { d.e. } \\
\text { c.v. }\end{array}$ & $\begin{array}{c}0,581 \\
0,126 \\
22 \\
\end{array}$ & $\begin{array}{c}259,8 \\
17,5 \\
7 \\
\end{array}$ & $\begin{array}{c}0,776 \\
0,087 \\
11 \\
\end{array}$ & $\begin{array}{c}0,852 \\
0,223 \\
26 \\
\end{array}$ & $\begin{array}{c}227,6 \\
29,3 \\
13 \\
\end{array}$ & $\begin{array}{c}0,733 \\
0,184 \\
25\end{array}$ \\
\hline Metopium brownei & $\begin{array}{l}\text { Media } \\
\text { d.e. } \\
\text { c.v. }\end{array}$ & $\begin{array}{c}0,377 \\
0,071 \\
19\end{array}$ & $\begin{array}{c}221,3 \\
17,1 \\
8\end{array}$ & $\begin{array}{c}0,429 \\
0,130 \\
30\end{array}$ & $\begin{array}{c}0,446 \\
0,094 \\
20\end{array}$ & $\begin{array}{c}170,6 \\
63,4 \\
37\end{array}$ & $\begin{array}{c}0,296 \\
0,07 \\
25\end{array}$ \\
\hline Bucida buceras & $\begin{array}{l}\text { Media } \\
\text { d.e. } \\
\text { c.v. }\end{array}$ & $\begin{array}{c}0,537 \\
0,096 \\
18\end{array}$ & $\begin{array}{c}245,9 \\
26,6 \\
11\end{array}$ & $\begin{array}{c}0,556 \\
0,087 \\
16\end{array}$ & $\begin{array}{c}0,707 \\
0,138 \\
20\end{array}$ & $\begin{array}{c}179,5 \\
37,9 \\
21\end{array}$ & $\begin{array}{c}0,497 \\
0,106 \\
21\end{array}$ \\
\hline
\end{tabular}


Tabla 4. Resultados Tenacidad por Impacto de 16 especies mexicanas

\begin{tabular}{|c|c|c|c|c|c|}
\hline \multirow[t]{2}{*}{ ESPECIE } & \multirow[t]{2}{*}{$\begin{array}{l}\text { VAL. } \\
\text { EST. }\end{array}$} & \multirow[t]{2}{*}{$\begin{array}{c}\text { DR } \\
\mathrm{pa} / \mathrm{vv}\end{array}$} & \multirow[t]{2}{*}{$\begin{array}{c}\mathrm{CH} \\
\%\end{array}$} & \multicolumn{2}{|c|}{$\begin{array}{c}\text { TENACIDAD } \\
\mathrm{kg}-\mathrm{cm} / \mathrm{cm}^{3}\end{array}$} \\
\hline & & & & seca & "verde" \\
\hline \multirow{3}{*}{ Cedrela odorata } & Media & 0,344 & 15,2 & 1,004 & 1,012 \\
\hline & d.e. & 0,068 & & 0,166 & 0,195 \\
\hline & C.v. & 20 & & 16 & 19 \\
\hline \multirow{3}{*}{ Lysiloma acapulcensis } & Media & 0,439 & 13,2 & 1,994 & 2,187 \\
\hline & d.e. & 0,049 & & 0,734 & 0,885 \\
\hline & c.v. & 11 & & 37 & 40 \\
\hline \multirow{3}{*}{ Guarea chichon } & Media & 0,500 & 13,1 & 1,840 & 1,820 \\
\hline & d.e. & 0,029 & & 0,597 & 0,495 \\
\hline & C.v. & 6 & & 32 & 27 \\
\hline \multirow{3}{*}{ Magnolia schideana } & Media & 0,540 & 13,8 & 2,840 & 3,358 \\
\hline & d.e. & 0,034 & & 0,402 & 0,940 \\
\hline & c.v. & 6 & & 14 & 28 \\
\hline \multirow{3}{*}{ Vatairea Iundellii } & Media & 0,547 & 13,6 & 2,506 & 1,948 \\
\hline & d.e. & 0,034 & & 0,599 & 0,543 \\
\hline & c.v. & 6 & & 24 & 28 \\
\hline \multirow{3}{*}{ Quercus eugeniifolao } & Media & 0,576 & 13,0 & 3,010 & 3,363 \\
\hline & d.e. & 0,038 & & 0,199 & 0,671 \\
\hline & C.V. & 7 & & 7 & 20 \\
\hline \multirow{3}{*}{ Getarda combsii } & Media & 0,579 & 13,1 & 2,654 & 2,999 \\
\hline & d.e. & 0,036 & & 0,345 & 0,814 \\
\hline & c.v. & 6 & & 13 & 27 \\
\hline \multirow{3}{*}{ Quercus uxoris } & Media & 0,583 & 12,8 & 2,949 & 2,774 \\
\hline & d.e. & 0,014 & & 0,267 & 0,450 \\
\hline & c.v. & 2 & & 9 & 16 \\
\hline \multirow{3}{*}{ Cosmocalyx spectabilis } & Media & 0,609 & 13,6 & 2,199 & 2,431 \\
\hline & d.e. & 0,061 & & 0,481 & 0,817 \\
\hline & C.V. & 10 & & 22 & 34 \\
\hline \multirow{3}{*}{ Quercus mexicana } & Media & 0,618 & 13,0 & 4,491 & 5,017 \\
\hline & d.e. & 0,027 & & 1,179 & 1,133 \\
\hline & c.v. & 4 & & 26 & 23 \\
\hline \multirow{3}{*}{ Lysiloma bahamense } & Media & 0,618 & 14,6 & 4,668 & 4,849 \\
\hline & d.e. & 0,064 & & 0,797 & 0,976 \\
\hline & c.v. & 10 & & 17 & 20 \\
\hline \multirow{3}{*}{ Ampelocera hottlei } & Media & 0,637 & 11,7 & 3,187 & 3,213 \\
\hline & d.e. & 0,030 & & 0,644 & 0,720 \\
\hline & C.V. & 5 & & 20 & 22 \\
\hline \multirow{3}{*}{ Mirandaceltis monoica } & Media & 0,684 & 13,4 & 2,585 & 2,561 \\
\hline & d.e. & 0,036 & & 0,714 & 0,610 \\
\hline & C.V. & 5 & & 28 & 24 \\
\hline \multirow{3}{*}{ Lonchocarpus castilloi } & Media & 0,753 & 15,9 & 4,366 & 5,001 \\
\hline & d.e. & 0,039 & & 1,904 & 1,344 \\
\hline & C.v. & 5 & & 44 & 27 \\
\hline \multirow{3}{*}{ Metopium brownei } & Media & 0,799 & 16,0 & 2,795 & 3,606 \\
\hline & d.e. & 0,073 & & 0,475 & 0,116 \\
\hline & C.V. & 9 & & 26 & 3 \\
\hline \multirow{3}{*}{ Bucida buceras } & Media & 0,828 & 15,6 & 4,230 & 5,641 \\
\hline & d.e. & 0,048 & & 1,518 & 0,745 \\
\hline & C.V. & 6 & & 36 & 13 \\
\hline
\end{tabular}




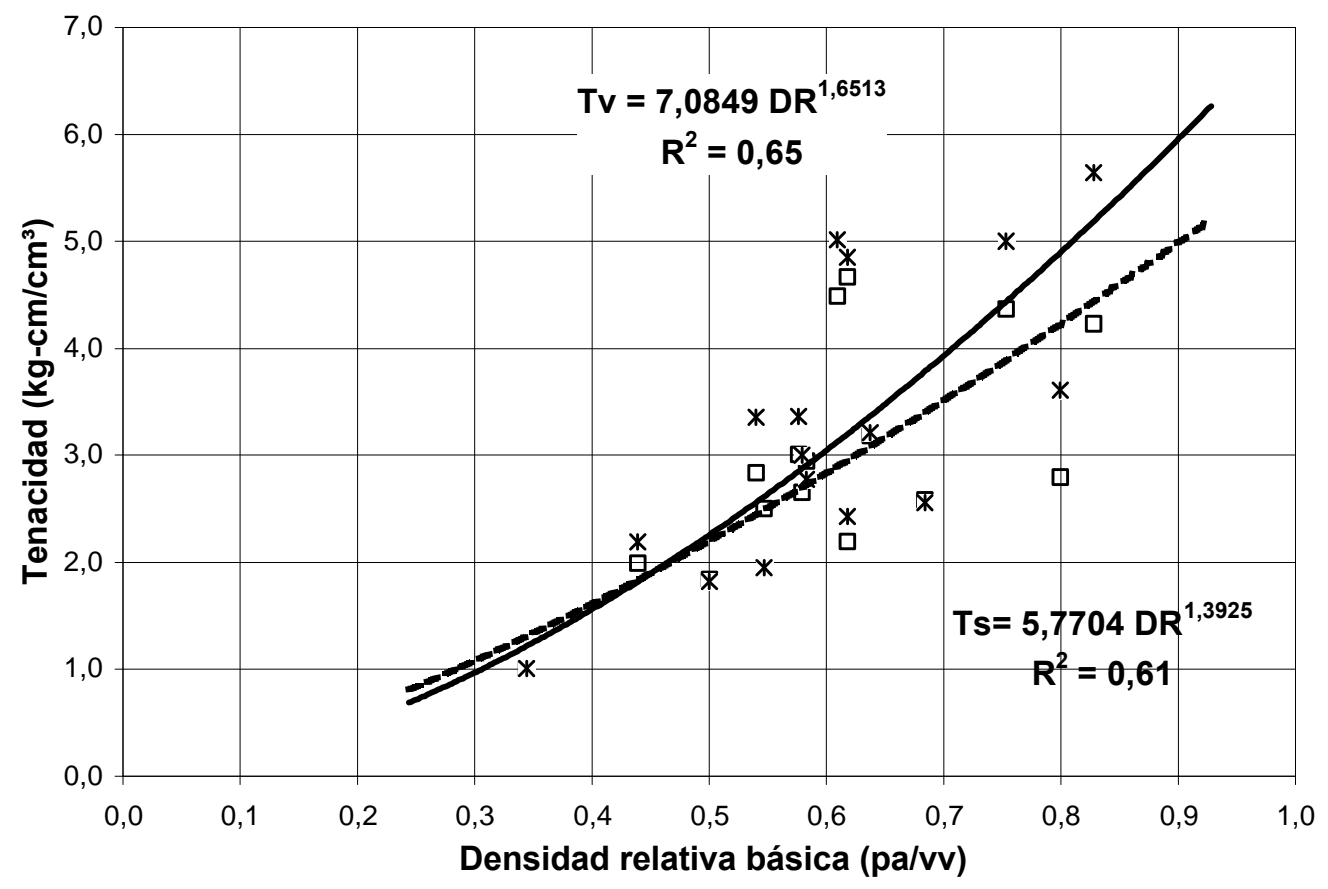

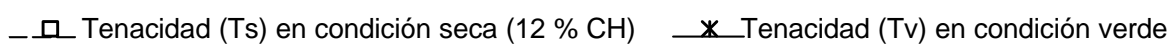

Figura 5. Valores promedio de tenacidad ( $\mathrm{T}$ ) en condiciones verde y seca para 16 maderas mexicanas graficadas contra su densidad relativa básica (Norma ASTM D143).

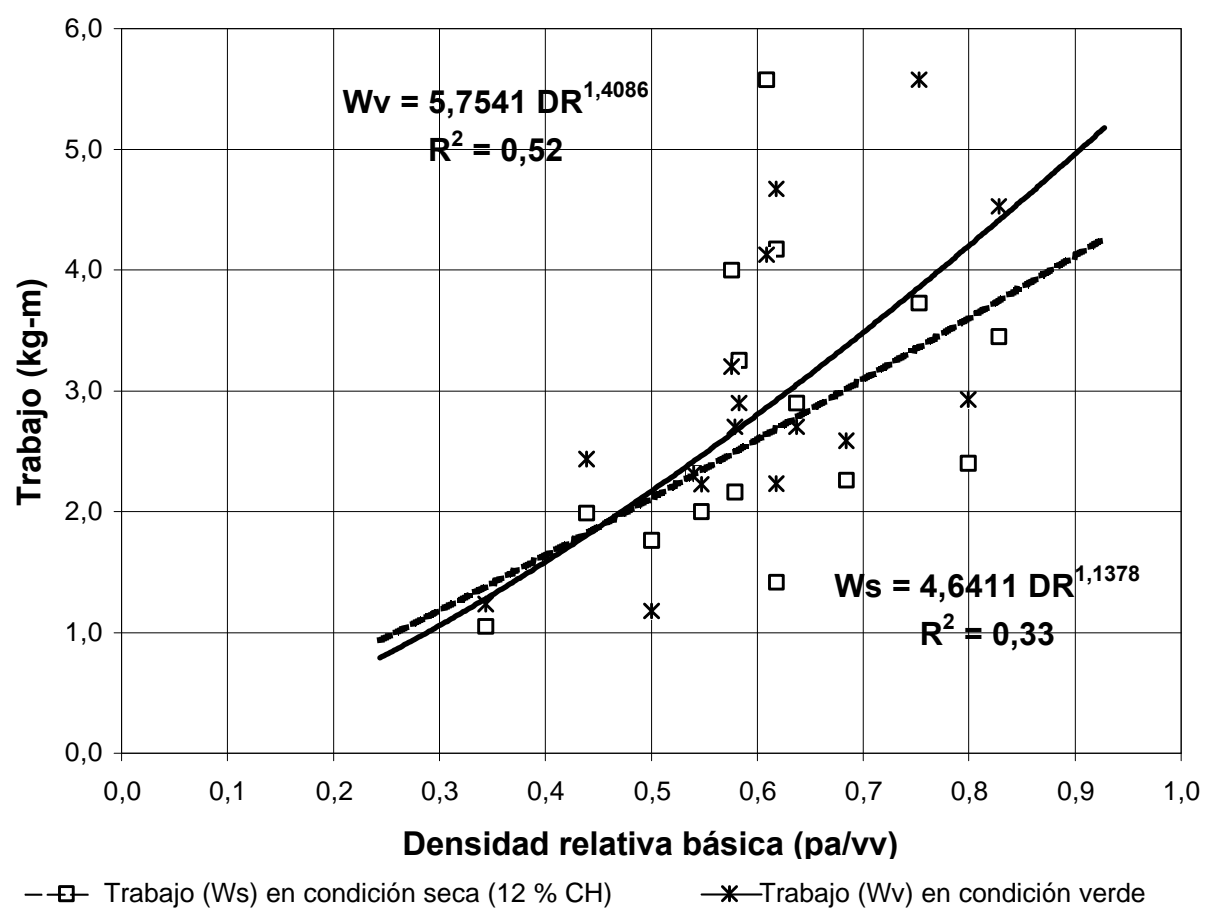

Figura 6. Valores promedio de trabajo (W) en condiciones verde y seca para 16 maderas mexicanas graficadas contra su densidad relativa básica (Norma NF B51-009). 


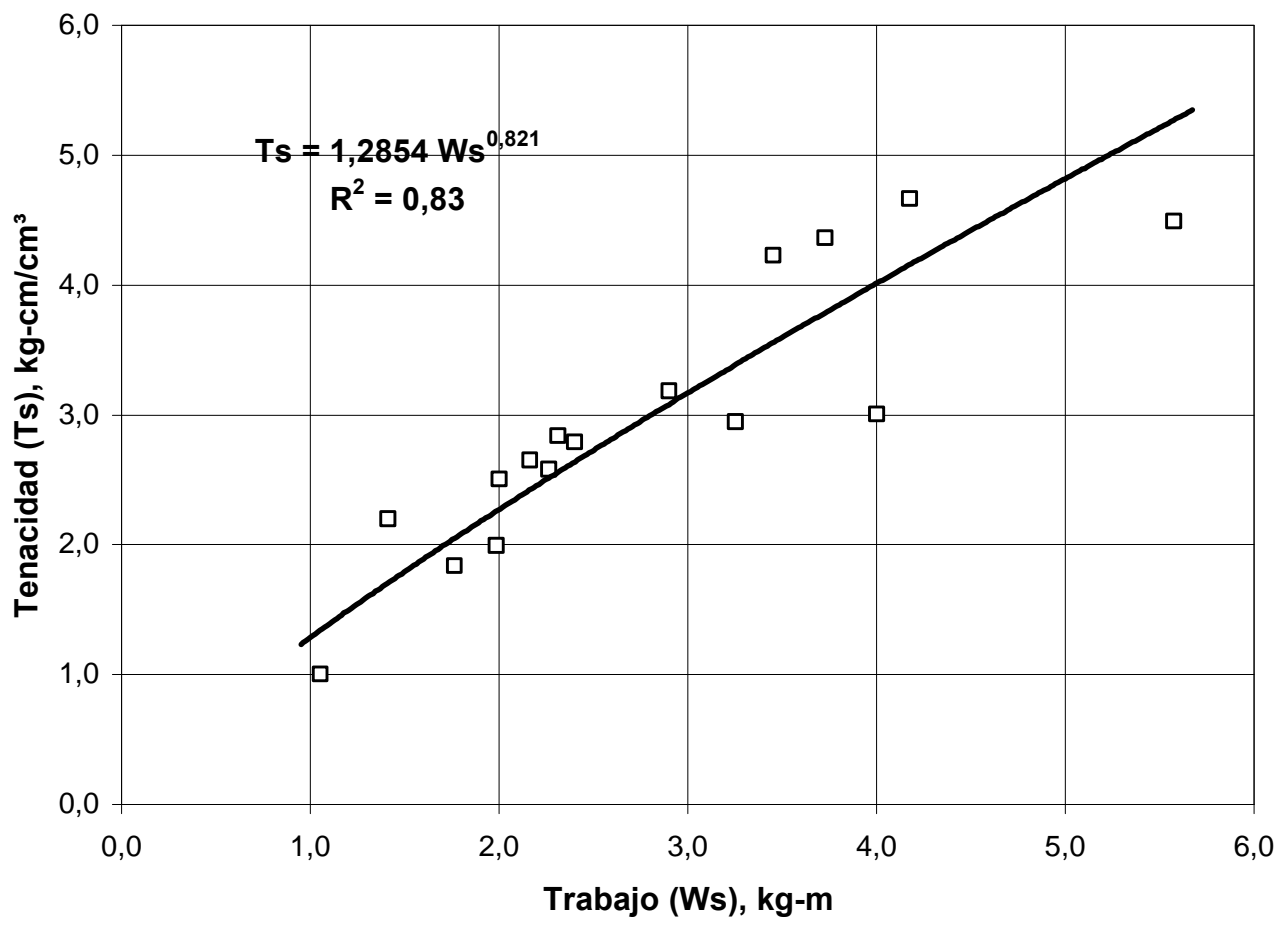

Figura 7. Correlación de valores de Trabajo (Ws) vs valores de Tenacidad (Ts) para 16 maderas mexicanas en condición seca.

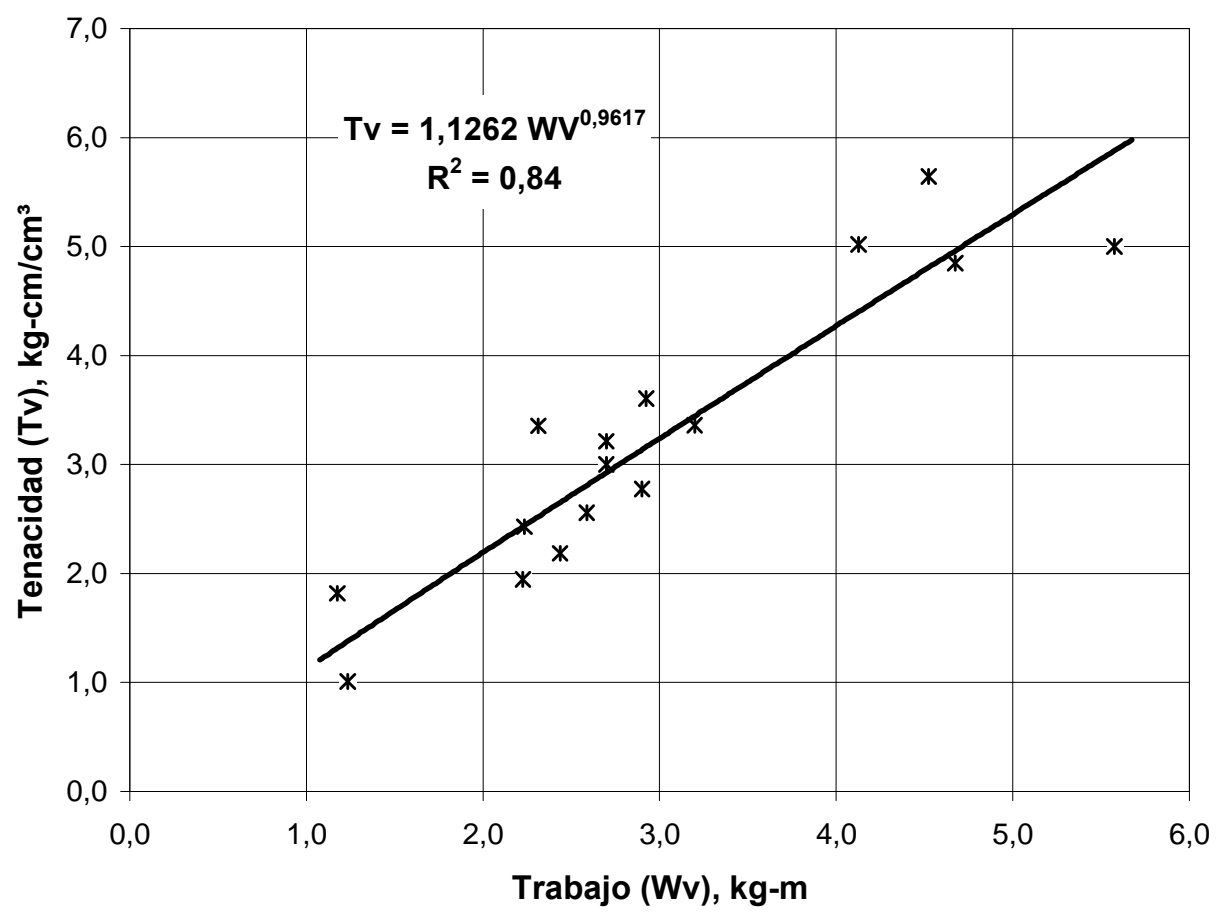

Figura 8. Correlación de valores de Trabajo (Wv) vs valores de Tenacidad (Tv) para 16 maderas mexicanas en condición verde. 
$\mathrm{CH}$, cuando éste es inferior al punto de saturación de la fibra, de acuerdo con lo mencionado por Echenique y Robles (1993).

Kollman (1968) citado por Christiansen (1997) menciona la evidencia de que la variaciones del contenido de humedad tiene un efecto muy ligero sobre la tenacidad. Christiansen (1997) también concluye que las variaciones encontradas por él en la tenacidad no son atribuibles completamente a las variaciones del contenido de humedad. En los datos de tenacidad publicados para maderas que crecen en Estado Unidos tampoco hay un efecto marcado sobre esta propiedad por el contenido de humedad (FPL, 1999).

En la dirección de aplicación de la carga: la carga en cada método de prueba se aplica en distinta dirección con respecto a los anillos de crecimiento. En el método basado en la Norma NF la carga se aplica en la dirección tangencial y en el método basado en la Norma ASTM la carga se aplica en la dirección radial. La madera presenta en general una mayor resistencia a las cargas por impacto en la dirección tangencial que en la radial (FPL, 1999).

Lo anterior hace necesario la realización de un estudio más profundo sobre el efecto del contenido de humedad y la dirección de aplicación de carga, así como su relación con la densidad relativa básica con el fin de generar información más precisa sobre esta propiedad para las maderas mexicanas

\section{REFERENCIAS}

ASTM (American Society for Testing and Materials). 1999. Standard methods for testing small clear specimens of timber. ASTM D143-99. Philadelphia, PA.

Barcenas P., G.M. 1995. Caracterización tecnológica de veinte especies maderables de la Selva Lacandona. Madera y Bosques 1(1):9-38.

Bárcenas P., G.M. y F. Ortega E. 1993. Fichas tecnológicas de cuatro especies de madera del boque mesófilo de montaña. Revista Forestal Latinoamericana 13/93:41-71.

Bodig. J. y B.A. Jayne. 1982. Mechanics of Wood and Wood Composites. Van Nostrand Reinhold Company. Nueva York. 712 p.

Brown, H.P.; A.J. Panshin y C.C. Forsaith. 1952. Text book of Wood Technology Vol II. Edit. McGraw-Hill. Nueva York. $783 \mathrm{p}$.

Christiansen, A.W. 1997. Effect of overdrying on toughness of yellowpoplar veneer. Holz al Roh-und Werkstoff 55(1997):71-75. SpringerVerlag.

Echenique M., R. y V. Díaz G. 1969. Algunas características tecnológicas de la madera de once especies mexicanas. Boletín Técnico No. 27. Secretaría de Agricultura y Recursos Hidráulicos. Subsecretaría Forestal y de la Fauna. Inst. de Investigaciones Forestales. p:16 y 35.

Echenique. M., R. y F. Robles F.V. 1993. Ciencia y tecnología de la Madera I. Textos Universitarios, Universidad Veracruzana.137 p.

FPL (Forest Products Laboratory). 1999. Wood handbook. Wood a Engineering material. United States Department of Agriculture Forest Service. General Technical Report FPL-GTR-113. Madison, Wi. 463 p.

Fuentes L., M.E. 1990. Propiedades físicomecánicas de cinco especies de encino (Quercus spp.) del estado de Puebla. Tesis Maestría. División de Ciencias Forestales Universidad Autónoma Chapingo. México. 52 p. 
INECOL, 2000. Manual de procedimientos de pequeñas probetas libres de defectos. Manual Interno.

Machuca V., R. 1995. Estudio tecnológico de la madera de Quercus insignis de Huatusco, Veracruz, México. Tesis de Licenciatura. División de Ciencias Forestales. Universidad Autónoma de Chapingo. $144 \mathrm{p}$.

NFA (L'A Association Francaise de Normalization).1942. Norme Francaise, bois essai de choc ou flexion dynamique. NF B51-009. Paris.

Negrete L., J.L. 1970 Algunas características físicas y anatómicas de la madera de cuatro especies de encino (Quercus) del Edo. de Michoacán. División de Ciencias Forestales. Universidad Autónoma de Chapingo. $71 \mathrm{p}$.

Novelo G., G. 1964. Posibles aplicaciones de la madera de cuatro especies tropicales con base en sus propiedades físicas y mecánicas. Tesis. Escuela Nacional de Agricultura. Chapingo, Edo. de México.

Ordóñez C., V.R.; G.M. Bárcenas P. y A. Quiroz S. 1989. Características físico-mecánicas de la madera de diez especies de San Pablo Macuiltianguis, Oaxaca. Boletín Técnico La madera y su uso no. 21. Instituto de Ecología - UAM. Xalapa, Ver. 30 p.
Ordóñez C., V.R., A. Quiroz S. y R.P. Zárate M.. 1998. Propiedades mecánicas de laminados estructurales con madera de encino. Madera y Bosques 4(2):95-104.

Panshin, A.J. y C. DeZeeuw. 1980 Textbook of Wood Technology. Structure, Identification, Properties, and Uses of the commercial woods of the United States and Canada. Edition, Vol.1. McGraw-Hill Book Co. Nueva York. 722 p.

Quiñónez O., J.O. 1974. Características físicas y mecánicas de la madera de cinco especies mexicanas. Boletín Técnico No. 4. Secretaría de Agricultura y Recursos Hidráulicos. Subsecretaría Forestal y de la Fauna. Inst. Investigaciones Forestales. 21 p.

Romero A., C., C. De la Paz P.O y G. Corral L. 1982. Características anatómicas y físico-mecánicas de ocho especies de coníferas de Baja California Sur. Boletín Técnico No. 51. 2a. Ed. Secretaría de Agricultura y Recursos Hidráulicos. Subsecretaría Forestal y de la Fauna. Instituto de Investigaciones Forestales. 48 p.

Zárate M., R.P.; V.R. Ordóñez C. y J.L. Martínez C. 2001. Determinación de algunas propiedades físicas y mecánicas de Grevillea robusta A. Cunn. del estado de Veracruz. Madera y Bosques 7(1):57-69.»

1 Departamento de Productos Forestales y Conservación de Bosques. Instituto de Ecología, A.C. Apdo. Postal 63. km 2.5 carr. ant. a Coatepec no. 351, Congregación El Haya. 91070 Xalapa, Ver. México. c.e.: barcenas@ecologia.edu.mx.

2 INIFAP. Campo experimental San Martinito. Apdo. Postal 124. 7400 San Martín Texmelucan, Puebla. México.

Manuscrito recibido el 13 de diciembre de 2002.

Aceptado el 29 de abril de 2003.

Este documento se debe citar como:

Bárcenas P., G.; R.P. Zárate M.; V.R. Ordóñez C.; A. Guerrero B. y J.A. Honorato. S. 2003. Correlación de los resultados de impacto o tenacidad de 16 maderas mexicanas utilizando dos métodos de prueba. Madera y Bosques 9(1):55-70. 